\title{
Multiple site silicon-based probes for chronic recordings in freely moving rats: implantation, recording and histological verification
}

\author{
A. Bragin ${ }^{\mathrm{a}, \mathrm{c}, *}$, J. Hetke ${ }^{\mathrm{d}}$, C.L. Wilson ${ }^{\mathrm{a}, \mathrm{c}}$, D.J. Anderson ${ }^{\mathrm{d}}$, J. Engel Jr ${ }^{\mathrm{a}, \mathrm{b}, \mathrm{c}}$, \\ G. Buzsáki ${ }^{\text {e }}$ \\ a Department of Neurology, UCLA School of Medicine, 2147 RNRC, 710 Westwood Plaza, Los Angeles, CA 90095-1769, USA \\ ${ }^{\mathrm{b}}$ Department of Neurobiology, UCLA School of Medicine, 2147 RNRC, 710 Westwood Plaza, Los Angeles, CA 90095-1769, USA \\ c Brain Research Institute, UCLA School of Medicine, 2147 RNRC, 710 Westwood Plaza, Los Angeles, CA 90095-1769, USA \\ ${ }^{\mathrm{d}}$ Center for Neural Communication Technology, The University of Michigan, Ann Arbor, MI, USA \\ e Center for Molecular and Behavioral Neuroscience, Rutgers, The State University of New Jersey, Newark, NJ, USA
}

Received 30 September 1999; received in revised form 22 February 2000; accepted 23 February 2000

\begin{abstract}
This paper describes the procedure of assembling a miniature microdrive and silicon probe system for surgical implantation into the adult rat brain. Successful recordings of single and multiunit activity with parallel depth profiles of spontaneous and evoked field potentials are shown. The procedure for histological verification of the position of the silicon probe is described. (C) 2000 Elsevier Science B.V. All rights reserved.
\end{abstract}

Keywords: Silicon probes; Hippocampus; Neocortex; Rats; Unit activity

\section{Introduction}

A large part of our knowledge about the physiological function of the brain is based on studies of sequentially analyzed single site recordings. Although it has long been recognized that the computational power of complex neuronal networks cannot be fully recognized by studying the properties of single cells or activity of a few selected sites, experimental access to the emergent properties of cooperating neurons has largely been impossible until quite recently. Direct investigation of the temporal dynamics of neuronal populations can only be based on simultaneous observation of large neuronal aggregates (Buzsáki et al., 1992; Wilson and McNaughton, 1994).

The two critical requirements towards this goal are (1) placing a large number of recording electrodes in a small amount of tissue at the submillimeter range; and (2) doing this without significant tissue damage. These major requirements put constraints on wire electrodes due to the limited number of recording sites. Additional con-

\footnotetext{
*Corresponding author. Tel.: +1-310-7942149; fax: +1-3102068461

E-mail address: abragin@ucla.edu (A. Bragin)
}

straints are the number of lead wires, preamplifiers and the size of the head connector that could be carried by a behaving animal. Progress in this field has been accelerated by the development of silicon microtechnology-based multichannel recording devices (Wise and Najafi, 1991). In such silicon devices, the number of recording sites is defined lithographically using the technology of integrated circuits. To date, typical probe thickness is $5-15 \mu \mathrm{m}$ with shank widths as narrow as 15 $\mu \mathrm{m}$. The tissue displacement for a single shank is typically less than a single wire electrode, and each shank can carry many isolated recording sites. An added advantage of silicon technology is that virtually any two-dimensional probe shape can be fabricated, with sites positioned laterally (on separate shanks) or in depth to customize the site configuration for a given application.

Despite of numerous trials of silicon probes, the literature relating to silicon probes used successfully for chronic recording of local field potentials and unit activity in freely moving animals is limited to a few laboratories. This may reflect the greater technical complexity of silicon probe utilization compared to the relatively simple use wire bundles. 
In previous papers, the main attention was placed on descriptions of manufacturing and surgical procedure (see Hetke et al., 1993). Below, we describe in detail the procedures for silicon probe implantation, recording with moveable silicon probes and histological verification of the position of silicon probes.

\section{Material and methods}

\subsection{Parts}

\subsubsection{Silicon probe}

A description of the probe fabrication process has been described in detail elsewhere (Drake et al., 1988; Anderson et al., 1989) and will only be outlined here. First, boron is selectively diffused into a silicon wafer to provide isolation on each step, which will later define the shape and thickness of the probe. Lower dielectric layers of silicon dioxide, silicon nitride and silicon dioxide are next deposited using chemical vapor deposition. Polysilicon, the interconnect material, is deposited, patterned and then capped with upper dielectric layers as just described. Contact openings are made through the top dielectrics using a combination of wet and dry etching and then metal, gold for bond pads and iridium for recording and/or stimulation sites, is deposited and patterned using lift-off techniques. Next, the field dielectrics are removed using reactive ion

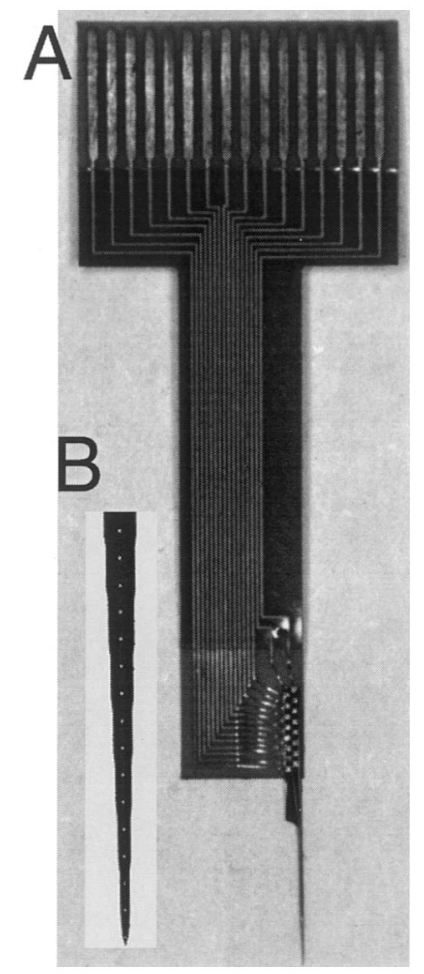

Fig. 1. Silicon probe wire bonded to flexible cable. Inset shows the silicon probe with a higher magnification. etching. Finally, the wafer is placed in ethylenediamine-pyrocatechol, which selectively etches the undoped silicon and leaves the remaining device materials unaffected.

\subsubsection{Printed circuitry board}

Because the connections to the circuitry of the silicon probe wafer are highly minimized a specially designed printed circuitry board (PC-board) was developed for wire bonding to silicon probes (Fig. 3). The PC board contains 16 holes, which match with an SMS-108-01GS ('Samtec', USA) connector matrix for acute experiments, or it can be soldered with individual wires to the pin connectors for chronic experiments (GM4 sockets glued together; Microtech, USA; Fig. 1).

\subsubsection{Flexible cable}

Another type of interface between the preamplifier and the silicon probe is the flexible printed cable. Similar to the PC board, the silicon probe is wirebonded to one end of the flexible cable. The other end of the cable contains soldering pads which are soldered to the pins of a high density ( $0.6 \mathrm{~mm}$ pin distance) connector matrix (Omnetics, USA). The flexible cables have several advantages. Less soldering required, the assembly occupies less space and is lighter than PC boards. However, they cost more and each probe and electrode arrangement requires new design and fabrication.

\subsubsection{Microdrive}

In order to move the probe within the brain a microdrive is fabricated from bronze channeling with cross section of $3 \mathrm{~mm}$ (Small Parts, Inc., USA). The $1.2-1.8-\mathrm{mm}$ stainless steel screw with the thread flattened at the end and a moveable platform in the middle is fixed in the center of the channel (Fig. 2A and B).

\subsection{Assembling}

The probe is attached to the PC board (or flexible cable) with epoxy. An ultrasonic bonder is then used to electrically link the probe bond pads to the copper traces on the PC board (or flexible cable) with $25 \mu \mathrm{m}$ aluminium wire. Exposed connections are then insulated using a combination of silicon and epoxy.

For soldering the PC-board to the individual wires, the PC-board with the wire-bonded silicon probe was fixed by double-coated Scotch tape on a small platform with the recording sites down. Under the dissecting microscope the holes of the PC-board were filled with solder up to the level of the PC-board surface. The PC-board was then soldered to 16, 4-5-cm long, 36 gauge Belden magnet wires (Newark Electronics) with the first $1.0 \mathrm{~mm}$ of insulation removed from the tips. After soldering, the wires were bent, arranged into a 
(A)
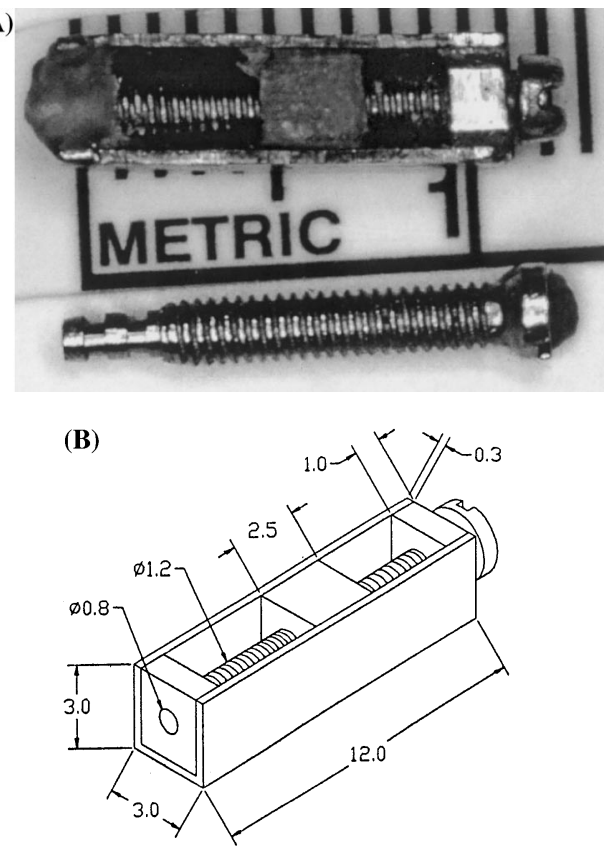

Fig. 2. (A) Picture of the assembled microdrive (top) and the screw that holds the movable platform; (B) schematic view of the microdrive shown in A.

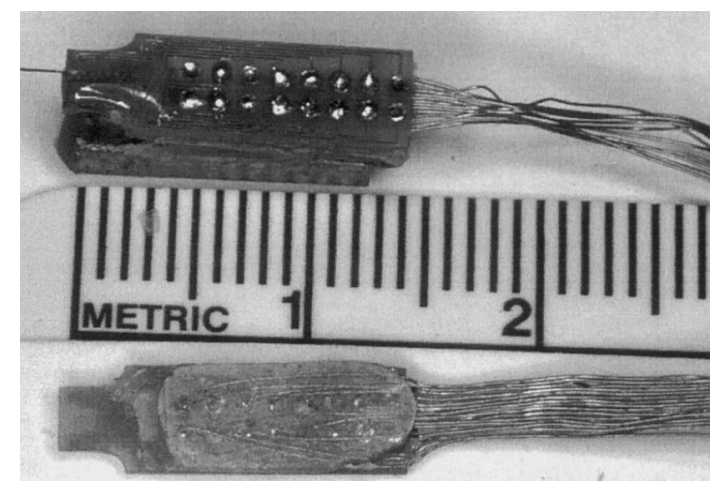

Fig. 3. The silicon probe assembled on the microdrive with the cable made from individually soldered wires and connectors (top) and the back part of the PC board with soldered wires covered by dental cement.

flat cable along the surface of the PC-board (Fig. 3) and covered with a thin layer of dental cement. The other ends of the wires were soldered to the output connectors (GM4 sockets; Microtech., USA).

After the cement dried, the silicon probe was carefully glued with dental cement or super glue to the microdrive, making sure that the tip of the probe was parallel to the longitudinal axis of the drive. After assembling the probe and microdrive, the assembly was gently rinsed with alcohol and kept in a plastic box humidified with alcohol on a cotton pledget inside the box until the time of surgery.

\subsection{Surgery}

For probe implantation rats were anesthetized with a mixture of ketamine $(100 \mathrm{mg} / \mathrm{kg})$, xylazine $(5.2 \mathrm{mg} / \mathrm{kg})$ and acepromazine $(1.0 \mathrm{mg} / \mathrm{kg})$ and fixed in the stereotactic frame. After the skull was exposed and cleaned, a hole with a diameter of $2 \mathrm{~mm}$ and coordinates $\mathrm{AP}=-3.5, \quad L=2.0$ was drilled over the hippocampus. Dura mater was gently removed, and the silicon probe was implanted in such a way that the recording contacts were inserted into the neocortex. For recording from neocortex, the silicon probe should just touch the surface of the neocortex until electrophysiological experiments start. The hole was covered with a warm $\left(\sim 50^{\circ} \mathrm{C}\right)$ mixture of mineral oil (one part) and dental wax (three parts). Stimulating electrodes were implanted into the perforant path and/or commissural pathways for determination of the position of the recording sites.

\subsection{Electrophysiological experiments}

Recordings began after a 3-4-day recovery period. Four-channel MOSFET input operational amplifiers (Buzsáki et al., 1989) or a 32-channel integrated amplifier (BAK Electronics, USA), mounted in the female connector served to eliminate cable movement artifacts. During the recording session, the probe was slowly moved down by turning the screw of the electrode carrier in steps of $1 / 4$ or $1 / 8$ of a turn $(90$ or $45 \mu \mathrm{m})$. As the silicon probe was advanced, single shocks of electrical pulses were delivered to the electrodes residing in the perforant or commissural pathways to determine the position of the probe within the hippocampus by evaluating the depth profile of the field responses (Andersen et al., 1971). Stimulation of thalamic relay nuclei or symmetric homotopic areas of contralateral neocortex can be used for identification of the position of the recording sites in the neocortex (Kandel and Buzsáki, 1997). After penetration of a cell layer the probe was either moved further down to record cells from other layers or retracted above the CA1 pyramidal layer, if it was necessary to collect more unit activity from the same area in subsequent experiments. After some days of rest the electrode was advanced again for further cell isolation and/or field potential recording.

\subsection{Perfusion and fixation}

After completion of electrophysiological experiments, rats are given an overdose of nembutal and perfused through the heart with saline $(100-200 \mathrm{ml})$ followed by $4 \%$ para-formaldehyde $(500-600 \mathrm{ml})$. The rats were then left at $4^{\circ} \mathrm{C}$ for $12-24 \mathrm{~h}$ to harden the brain. The brain was removed under a surgical microscope. The wax covering the hole was gently removed to expose the 
silicon probe, which was cut with microsurgical scissors near the surface of the brain. Then the drive, dental cement and bone were removed. Special attention was paid to remove the dura mater that may adhere to the silicon probe and pull it from the brain. After removal from the skull, the brain with the tip of the silicon probe still in place was left in the fixative for several days before cutting.

\subsection{Brain sectioning and staining}

After 3-7 days of fixation, the brain was sectioned in a vibratome. The caudal part of the brain was aligned parallel to the silicon probe by visual estimation under a surgical microscope and glued to the platform. The

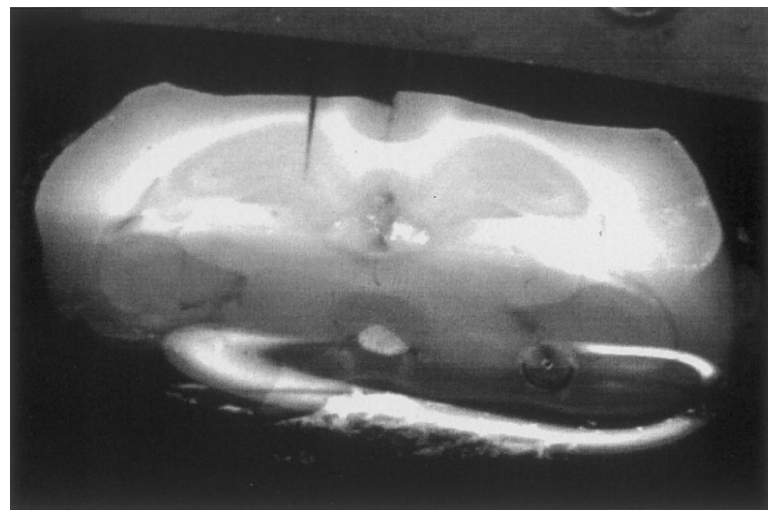

Fig. 4. Picture of the brain during vibratome sectioning when the silicon probe appears in the upper part of the hippocampus.

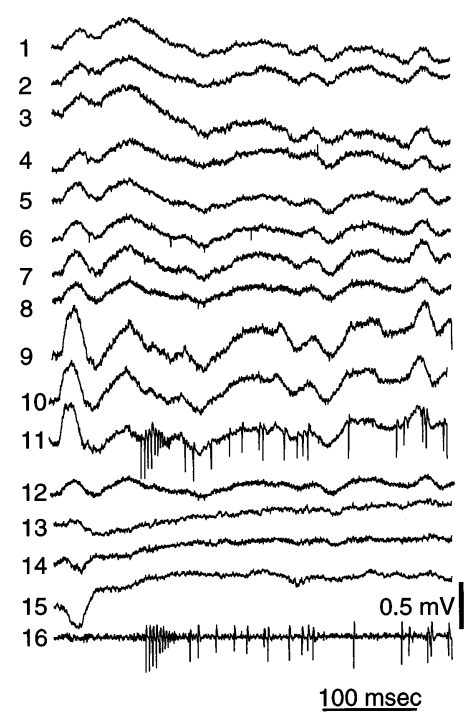

Fig. 5. An example of local field potentials and unit activity recorded with the silicon probe. Local field potentials are shown on the left part of figure with the inversion of the signal at channels 14-15. Unit activity with signal to noise ratio at more than $5: 1$ is present in recording site \# 11; other units with 3:1 and 2:1 signal to noise ratios are visible in recording sites \#6 and \#7. Channel 16 demonstrates the same unit activity of channel 11 after high pass filtering. platform was fixed in such a way that the blade cut the brain parallel to the position of the silicon probe. Sections cut at $50-60 \mu \mathrm{m}$ were used in order to allow the silicon probe to remain intact within one of the sections. It is useful to photograph the block during sectioning (Fig. 4), so that if the plane of cutting is not perfectly parallel, the position of the silicon probe can be closely estimated if it is lost during vibratome sectioning. When the brain slice containing the silicon probe was cut, it was mounted on a gelatin-coated slide and left to dry for 12-24 h. Nissl or other staining was carried out without shaking the slide during the staining procedure.

\section{Results}

The unit recording with silicon probe can be obtained routinely and these results in freely moving rats have been described in many earlier publications (Buzsáki et al., 1992; Bragin et al., 1995, 1996, 1997; Ylinen et al., 1995; Chrobak and Buzsáki, 1996, 1998; Kandel and Buzsáki, 1997). See also list of publications in homepages http://osiris.rutgers.edu and www.engin. umich.edu/facility/cnct/papers.html.

A special advantage of simultaneous sampling of field potentials is that current-source calculations can be performed on-line and, instead of voltage traces, the behavior-dependent changes of sinks and sources can be monitored continuously (Ylinen et al., 1995; Bragin et al., 1997; Kandel and Buzsáki, 1997; Castro-Alamancos, 1999). Field experiments were typically carried out with 16-site silicon probes (Fig. 5). Unit activity was recorded simultaneously and continuously with the field. Recording sites residing in cell body layers provide units with very large signal-to-noise ratios. When positioned in the cell layer, units were typically present on several shanks although probability of unit recording was higher from lower recording sites compared with upper recording sites.

An advantage of the linear arrangement of the recording tips is that action potentials generated by the soma and dendrites can be monitored simultaneously, provided that the recording sites are aligned with the apical dendrite (Buzsáki and Kandel, 1998; Buzsáki et al., 1992). However, because of the distance between the recording sites $(100 \mu \mathrm{m})$, simultaneous recording from the same cell(s) was not possible in all structures.

The recording sites can be determined in vivo by monitoring unit activity and the amplitude versus depth distribution of spontaneous and evoked field potentials. A typical depth profile of evoked potentials in response to perforant path stimulation in the CA1-dentage gyrus axis is shown in Fig. 6. Nevertheless, determination of the mediolateral position of the electrode requires subsequent histological analysis. Although the recording 
A

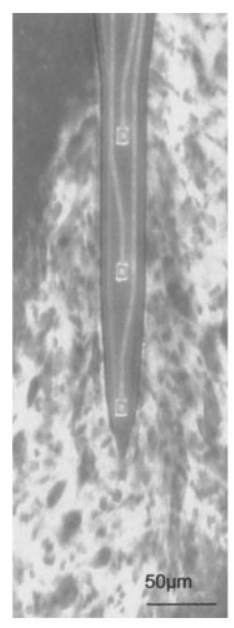

B

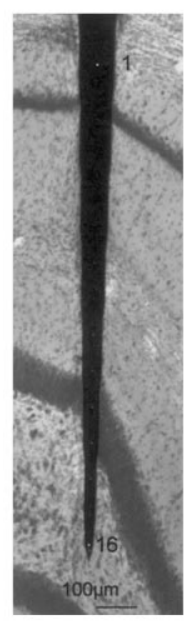

C

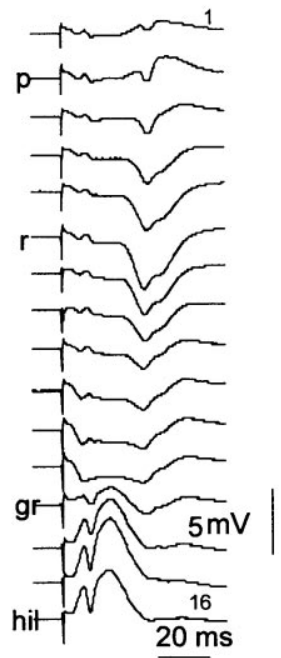

Fig. 6. (A) Detail of the tip of the silicon probe shown in B magnified to illustrate the platinum-iridium electrode contacts spaced at 100$\mu \mathrm{m}$ intervals; (B) hippocampal 60- $\mu \mathrm{m}$ Nissl-stained section containing the same silicon probe viewed from the back; (C) depth profiles of field potentials evoked by commissural stimulation recorded with the silicon probe shown in $\mathrm{B}$.

track can be recovered routinely in each experiment, we have tested various ways of preserving the probe in situ.

In five rats during cutting, brains were oriented in such a way that the silicon probes were perpendicular to the vibratome's blade. In all cases the silicon probe was moved away from the original point of recording for a distance $200 \mu \mathrm{m}$ or more. In four cases silicon probe during cutting was oriented parallel to the blade and in all of these experiments it did not move during cutting for more than $50 \mu \mathrm{m}$ from the original point of recording. Fig. 6B shows an example of a silicon probe implanted in the dorsal hippocampus after sectioning and Nissl staining. The track of the silicon probe is visible. In the illustrated case the silicon probe during cutting moved less than $100 \mu \mathrm{m}$ from the original position in the brain during electrophysiological experiments. Fig. 6A demonstrates the position the tip of the same silicon probe under higher magnification, with the microphotograph made from the opposite side of the section. The exact position of the probe, relative to cellular and dendritic layers, can also be estimated on the basis of the shape of evoked potentials in response to electrical stimulation of the hippocampal afferents. Fig. 6C shows a voltage versus depth profile of the responses to commissural pathway stimulation.

Different probe configurations have been used for recording field potentials and unit activity in the hippocampus, thalamus, entorhinal cortex and neocortex. For recording from deep structures, such as the entorhinal cortex, silicon probes with ultra-long shanks (15 mm) have been used (Chrobak and Buzsáki, 1996).
Unit separation based on 'stereotrode' and 'tetrodes' algorithms (McNaughton et al., 1983; Gray et al., 1995) is not always possible. Spike separation with single units linear 'tetrodes' or 'hexatrodes' with $25 \mu \mathrm{m}$ vertical tip separation has more advantages because it gives better representation of multiunit activity in the threedimensional space. When the 16-site probe is moved through a cell body layer (e.g. CA1 pyramidal layer), the same sets of neurons can be recorded by the successive recording sites of the probe. The signal-to-noise ratio begins to deteriorate when approximately three quarters of the probe have already penetrated the layer (i.e. recording site 4 and above in Fig. 6). This is due to the widening of the shank $(>60 \mu \mathrm{m})$. Nevertheless, multiple units can still be recorded from sites 1 and 3, although their amplitude is less when compared to the same site recorded by sites located at the lower half of the probe. Two to six shanks with 150 or $300 \mu \mathrm{m}$ shank separation moved by a single separate microdrive allow further investigation-communication between the local circuitries.

\section{Conclusion}

Although the procedure of preparation and verification of the position of the silicon probe is time consuming, it provides great advantages for analysis of brain circuitry in freely moving animals. It also allows analysis of voltage depth profiles of spontaneous and evoked potentials with simultaneous recordings from sites with fixed separation spaces between the recording points. The precise identification of the position of the recording sites with verification on histological sections allows analysis of local interactions between neuronal elements of given networks.

\section{Acknowledgements}

The authors thank Maria Melendez, Erick and Gena Behnke, Tatyana Fox for their help in the manuscript preparation. This work was supported by NIH and NIH/NCRR grants (NS34994; MH54671; NS33310; P41RRO9754).

\section{References}

Andersen P, Bliss TVP, Skrede KK. Lamellar organization of hippocampal pathways. Exp Brain Res 1971;13:222-38.

Anderson DJ, Najafi K, Tanghe SJ, Evants DA, Levy KL, Hetke JF, Xue X, Zappia JJ, Wise KD. Bath-fabricated thin-film electrodes for stimulation of the central auditory system. IEEE Trans Biomed Eng 1989;36:693-704.

Bragin A, Jando G, Nadásdy Z, Hetke J, Wise K, Buzsáki G. Gamma $(40-100 \mathrm{~Hz})$ oscillations in the hippocampus of the behaving rat. J Neurosci 1995;15:47-60. 
Bragin A, Csicsvari J, Penttonen M, Penttonen G, Buzsáki B. Epileptic afterdischarge in the hippocampal-entorhinal system: current source density and unit studies. Neuroscience 1997;76:1187-203.

Buzsáki G, Kandel A. Somadendritic backpropagation of action potentials in cortical pyramidal cells of the awakened rat. J Neurophysiol 1998;79:1587-91.

Buzsáki G, Bickford RG, Ryan LJ, Young S, Prohaska O, Mandel RJ, Gage FH. Multisite recording of brain field potentials and unit activity in freely moving rats. J Neurosci Methods 1989;28:209-17.

Buzsáki G, Horvath Z, Urioste R, Hetke J, Wise K. High frequency network oscillation in the hippocampus. Science 1992;256:1025-7.

Castro-Alamancos MA. Neocortical synchronized oscillations induced by thalamic disinhibition in vivo. J Neurosci 1999;19:1-7.

Drake KL, Wise KD, Farraye J, Anderson DJ, BeMent SL. Performance of planar multisite microprobes in recording extracellular single-unit intracortical activity. IEEE Trans Biomed Eng 1988;35:719-32.

Chrobak J, Buzsáki G. High-frequency oscillations in the output networks of the hippocampal-entorhinal axis of the freely behaving rat. J Neurosci 1996;16:3056-66.
Chrobak J, Buzsáki G. Gamma oscillations in the entorhinal cortex of the freely behaving rat. J Neurosci 1998;18:388-98.

Gray CM, Maldonado PE, Wilson M, McNaughton B. Tetrodes markedly improve the reliability and yield of multiple single-unit isolation from multi-unit recordings in cat striate cortex. J Neurosci Methods 1995;63:43-54.

Hetke JK, Lund JL, Najafi K, Wise KW, Anderson DJ. Silicon ribbon cables for chronically implantable microelectrode arrays. IEEE Trans Biomed Eng 1993;41:314-21.

Kandel A, Buzsáki G. Cellular-synaptic generation of sleep spindles, spike-and-wave discharges, and evoked thalamocortical responses in the neocortex of the rat. J Neurosci 1997;17:6783-97.

McNaughton BL, Barnes CA, O'Keefe J. The contributions of position, direction, and velocity to single unit activity in the hippocampus of freely-moving rats. Exp Brain Res 1983;52:41-9.

Wilson MA, McNaughton BL. Reactivation of hippocampal ensemble memories during sleep. Science 1994;265:676-9.

Wise KD, Najafi K. Microfabrication techniques for integrated sensors and microsystems. Science 1991;254:1335-42.

Ylinen A, Bragin A, Nadásdy Z, Jando G, Szabo I, Sik A, Buzsáki G. Sharp wave-associated high-frequency oscillations $(200 \mathrm{~Hz})$ in the intact hippocampus: network and intracellular mechanisms. J Neurosci 1995;15:30-46. 